\title{
XXXIII. On Bernoulli's solution of the problem of shortest twilight
}

\author{
T.S. Davies Esq. F.R.SS.L. \& E. F.R.A.S.
}

To cite this article: T.S. Davies Esq. F.R.SS.L. \& E. F.R.A.S. (1833) XXXIII. On Bernoulli's solution of the problem of shortest twilight, Philosophical Magazine Series 3, 3:15, 179-185, DOI: 10.1080/14786443308648153

To link to this article: http://dx.doi.org/10.1080/14786443308648153

册 Published online: 01 Jun 2009.

Submit your article to this journal $₫$

Џ Article views: 2

Q View related articles $\sqsubset$ 


\section{$\left[\begin{array}{ll}179 & ]\end{array}\right.$}

XXXIII. On Bernoulli's Solution of the Problem of Shortest Twilight. ByT. S. Davies, Esq. F.R.SS. L. \& E., F.R.A.S., \&c.*

TTHE problem of the shortest twilight was first solved, and very elegantly too, by Nunez, in a little quarto tract of 14.2 pages, printed at Lisbon in 154.2; and it has engaged the attention of many distinguished authors since that time. John and James Bernoulli as well as D'Alembert and L'Hôpital discussed the problem: but the researches of the two illustrious brothers are nowhere, that I know of, to befound in print. The result is, indeed, stated in one of John Bernoulli's letters to the Journ. des Savans: but he says nothing that can lead us to discover the particular details of his solution. He however certainly bestowed a good deal of trouble upon it; as he says, " $J$ 'ai résolu le problême de trouver géométriquement le jour du plus petit crépuscule, ce qui a occupé mon frère, Professeur de Mathématique à Bâle, et moi, depuis plus de cinq ans, sans en pouvoir venir à bout." He tells us, too, that he effected his solution by means of the differential calculus. D'Alembert, in the Encyclopédie Méthodique (Art. CrépusCULE), undertook to give a complete solution on the supposed plan of Bernoulli : but he obtained also an equation of the fourth degree, the roots of which embarrassed him considerably. Two of the roots, it is true, he dismissed from his formula pretty readily; but not, as it appears to me, upon grounds altogether satisfactory. He does not attempt to show either how they came there or what was their actual signification; nor does he even attempt to do so with the remaining root of the remaining pair, though he employs more than three folio columns in showing (by special instead of general reasonings) that it does not belong to the minimum, and he even says, and attempts to prove, that it does not refer to the maximum twilight problem.

The intricacy of D'Alembert's solution seems to have deterred successive geometers from attempting to develop in a more advantageous form the probable method of Bernoulli, these being the only solutions (L'Hôpital's perhaps excepted, of which I cannot speak, not having his book within reach,) which $I$ have found, in which the modern method of maxima and minima is employed. Every other solution with which I am acquainted proceeds upon certain geometrical considerations derived from the nature of the figure itself rather than from the equations of the problem: and it must be admitted that in point of facility these have very considerably the ad-

- Communicazed by the Author.

$2 \mathrm{~A} 2$ 
vantage over the other method. Had, therefore, the mere solution of the problem been the object to be principally sought for, there can be no doubt that it is already rendered as simple as it ever can be ; and a very ample list of such solutions may be consulted in Professor Leybourn's edition of that valuable but unpretending little periodical, the Ladies' Diary, vol. iv. p.314. We find there the solutions of Messrs. Ivory, Wallace, and Skene, as well as the very amplified discussions of Delambre, as given both in his Astronomie, and in his Histoire de l'Astronomie Ancienne. Two other solutions by Hachette, printed in the Correspondence of the Polytechnic School, vol. ii.; and others still, by Keill, Lemonnier, Maupertuis, Emerson, Mauduit, Cagnoli, \&c., may be found in the respective writings of those authors. All these, however, are of the kind which may be called geometrical, in contradistinction to the method of Bernoulli and D'Alembert.

Of these, Mr. Skene's is the only one which takes the problem in the more general form, in which another almacantar is substituted for the horizon of the common problem; and his solution is truly elegant as a specimen of the method of research which he adopted. Unfortunately it confined him to the simple question of the minimum, and he also was thereby led to give an incomplete solution, compared with that furnished by the calculus. The two cases, the maximum and the minimum, are so intimately connected that they present themselves naturally as the extreme cases of the question, When the duration of trilight is given, to find the declination; and they appear together in the same final equation interwoven in a single formula, and demanding in all respects equal attention. On this account, elegant as the solution of Mr. Skene is, it does not remove the necessity for reconsidering the problem under another aspect.

Some time ago, when composing a paper (since published in vol. xii. of the Edinburgh Transactions,) on the application of great-circle co-ordinates to the investigation of spherical loci, I was led to examine a considerable number of those problems which had been subjects of discussion, by the older methods of treating spherical curves-and the problem of shortest twilight amongst the rest. As, however, that problem did not properly appertain to my plan, I relinquished the inquiry for others more interesting to me at that moment, and laid aside the few memoranda which $I$ had made concerning it. A day or two ago my attention was accidentally recalled to the subject; and as in those memoranda the problem had been viewed under its most general aspect, and the true character of all the roots of the equation assigned,-as, moreover, the 
problem is one of some historical celebrity, and its solution calculated to enforce an important truth,- on all these accounts its occupying a place in the Philosophical Magazine may perhaps be justified.

PROBLEM.-Let EHRQ be the horizon of the place; $\mathrm{Z}$ the zenith; $\mathrm{P}$ the pole; $\mathrm{ESQ}$ the equator; MUP', LNT any troo almacantars; LNPM a circle of declination; then it is required to find the position or polar distance of LNPM, so that the arc of it intercepted between the given almacantars may be a maximum or a minimum.

Such is the more general geometrical statement of the problem in the aspect under which the Bernoullis viewed it: the angle $\mathrm{NPP}^{\prime}$ is the measure of the time elapsed between the sun's passing the two almacantars. In the common problem for one of these almacantars is substituted the horizon itself.

Let $N^{\prime}$ be the intercepted portion of the circie of declination. Denote the colatitude, or PZ, by $\lambda$ : put $\rho_{,} \rho_{l}, \rho_{\| \prime}$ the spherical radii of the circles $L R N$, LTN, and MUP' respectively:

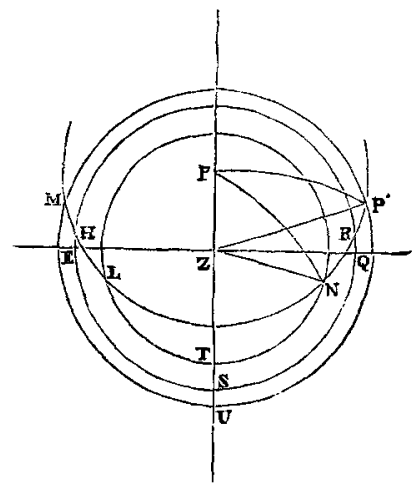
and denote the angles $\mathrm{ZPP} l$ and $\mathrm{ZPN}$ by $\theta_{\| /}$and $\theta_{i}$. Then;

$$
\begin{aligned}
& \theta_{1}=\cos ^{-1} \frac{\cos \rho_{1}-\cos \rho \cos \lambda}{\sin \rho \sin \lambda} \\
& \theta_{11}=\cos ^{-1} \frac{\cos \rho_{11}-\cos \rho \cos \lambda}{\sin \rho \sin \lambda}
\end{aligned}
$$

From which we have the polar angle or measure of the time,

$$
\begin{aligned}
& \theta_{l l}-\theta_{l}=\cos ^{1}-\frac{\cos \rho_{l l}-\cos \rho \cos \lambda}{\sin \rho \sin \lambda} \\
& -\cos 1-\frac{\cos \rho_{1}-\cos \rho \cos \lambda}{\sin \rho \sin \lambda}
\end{aligned}
$$

But that the time may be a maximum or a minimum, we must have

$$
\begin{aligned}
& d\left\{\cos ^{1-\frac{\cos \rho i}{11}-\cos \rho \cos \lambda}\right. \\
& \left.\cos ^{1}-\frac{\cos \rho_{1}-\cos \rho \cos \lambda}{\sin \rho \sin \lambda}\right\}=0 \ldots \ldots
\end{aligned}
$$


In this expression $g$ is the variable, and the differentiation being performed with respect to it, we have, after transposition,

$$
\begin{aligned}
& \frac{\left(\cos \lambda-\cos \rho_{1} \cos \rho\right) \operatorname{cosec} \rho}{\sqrt{\left(\sin ^{2} \lambda-\cos ^{2} \rho_{1}\right)+2 \cos \rho_{1} \cos \lambda \cos \rho-\cos ^{2} \rho}} \\
& =\frac{\left(\cos \lambda-\cos \rho_{11} \cos \rho\right) \operatorname{cosec} \rho}{\sqrt{\sin ^{2} \lambda-\cos ^{2} \rho_{11}+2 \cos \rho_{1} \cos \lambda \cos \rho-\cos ^{2} \rho}} \ldots \text { (3) }
\end{aligned}
$$

Squaring these to take the radical from the denominator, and transposing all to one side, it takes the form

$$
\begin{aligned}
0 & =\operatorname{cosec}^{2} \rho\left\{\frac{\left(\cos \lambda-\cos \rho_{1} \cos \rho\right)^{2}}{\sin ^{2} \lambda-\cos ^{2} \rho+2 \cos \lambda \cos \rho_{1} \cos \rho-\cos ^{2} \rho}\right. \\
& \left.-\frac{\left(\cos \lambda-\cos \rho_{11} \cos \rho\right)^{2}}{\sin ^{2} \lambda-\cos ^{2} \rho_{11}+2 \cos \lambda \cos \rho_{11} \cos \rho-\cos \rho^{2}}\right\} \ldots \ldots()
\end{aligned}
$$

This expression is probably not much unlike that which John Bernoulli obtained, allowance being made for the greater generality of its objects and the difference of the notations. As, however, he merely describes it, we can only conjecture what it might have been*: at all events it does not greatly differ from that given by D'Alembert, in the Encyclopédie Méthodique, Art. Crepuscule. Let us now attend to the reduction of the factor within the brackets : and first, cross multiply, and arrange the resulting terms according to the powers of $\cos \rho$; then divide all the terms by $\cos ^{2} \rho_{11}-\cos ^{2} \rho_{1}$ : our result is

$$
\begin{gathered}
\cos ^{4} \rho-2 \cos \lambda \frac{1+\cos \rho_{1} \cos \rho_{\|}}{\cos \rho_{1}+\cos \rho_{\| 1}} \cos ^{3} \rho-\sin ^{2} \lambda \cos ^{2} \rho \\
+2 \cos \lambda \frac{1+\cos \rho_{1} \cos \rho_{\| \prime}}{\cos \rho_{1}+\cos \rho_{\|}} \cos \rho-\cos ^{2} \lambda ;
\end{gathered}
$$

which may again be readily changed into

$$
\begin{aligned}
& \left(1-\sin ^{2} \rho\right)^{2}+2 \cos \lambda \cdot \frac{1+\cos \rho_{1} \cos \varphi_{\|}}{\cos \rho_{l}+\cos \varphi_{I 1}} \cdot \cos \rho\left(1-\cos ^{2} \rho\right) \\
& -\sin ^{2} \lambda\left(1-\sin ^{2} \rho\right)-\cos ^{2} \lambda ;
\end{aligned}
$$

and this is at once converted into

$$
\sin ^{2} \varrho\left\{\cos ^{2} \varrho-2 \cos \lambda \frac{1+\cos \rho_{1} \cos \rho_{11}}{\cos \rho_{1}+\cos \rho_{\| 1}} \cos \rho+\cos ^{2} \lambda\right\} \ldots \ldots \ldots
$$

Inserting instead of the bracketed factor of (4) its value given by (5), we have that equation (4) at once transformed irito

$$
\cos ^{2} \rho_{-}-2 \cos \lambda \frac{1+\cos \rho_{1} \cos \rho_{11}}{\cos \rho_{1}+\cos \rho_{u}} \cos \rho+\cos ^{2} \lambda=0
$$

* Jnhan. Bernoulli, Opera Omnia, tome i. p. 64. 
We thus see that the factor $\sin ^{2} \rho$ which arose out of the process of finding $(5)$ is really a foreign factor introduced, as those factors so generally are, by elimination : and we see too how it arose; viz. by Bernoulli and D'Alembert having cancelled the factor $\operatorname{cosec}^{z} \rho$ from equation (4) merely because it was a factor of the whole equation. Had this view occurred to them, they would have depressed their biquadratic to a quadratic at once, and have escaped the embarrassment created by attempting to prove that certain roots contained in it did not fulfill the conditions of the problem, and were therefore inadmissible. But an important question here arose-what is the signification of this result, and how did it make its appearance intermingled with the proper solution of the problem? It is not enough to take those parts of a result which we can readily interpret, and reject all the others as useless or unmeaning: though such is and always has been so greatly the fashion, that scarcely a single author has insisted upon an unflinching determination to consider every solution incomplete which stops short of this consummation. It will, I have no doubt, ere long, become a fundamental principle in the philosophy of mathematics-that every part of an algebraical result admits of complete interpretation, either by reference to the conditions which were expressed in the fundamental equations, or else in the hypotheses, tacitly made (in order to apply our transforming operations,) in the various subsequent stages of the solution. It is, however, so much the custom to discard, without consideration or remark, all such results as do not admit of a ready and obvious application to the immediate objects before us, that facility of interpretation is the rarest of all the faculties of the geometer that we find in any considerable degree developed. No factor ought to be rejected for which a satisfactory reason cannot be given, nor ought it to be considered foreign till the step at which it was introduced, is distinctly ascertained: and it bespeaks both a bad taste and unpardonably negligent habits of research, to leave any part of the final equation unexplained, even in the most trivial inquiry which may be undertaken*.

- The valuable discussion and illustrations of this principle, given by Mr. Babbage in vol. ii. of the Cambridge Philosophical Transactions, cannot be too earnestly recommended to every young mathematician who would form a proper taste in the modern analysis, nor too diligently studied by every one who aspires to a higher character than that of a mere algebraical machine.

With regard, however, to the metaphysical views entertained by that distinguished philosopher, on the relations between the mind and certain objects of mental action, as expressed in that paper, I offer no opinion here, as I shall have occasion to moot that subject in another place. 
Resolving now the quadratic equation (6), we have

$$
\begin{aligned}
& \cos \rho=\cos \lambda \frac{1+\cos \rho_{1} \cos \rho_{11} \pm \sin \rho_{1} \sin \rho_{\| \prime}}{\cos \rho_{1}+\cos \rho_{\| \prime}} \\
& =\cos \lambda \frac{1+\cos {\overline{\rho_{1} \tilde{+}}}_{\overline{\cos }}}{\cos \rho_{1}+\overline{\cos \rho_{I I}}} \\
& =\cos \lambda \frac{\cos ^{2} \frac{1}{2} \rho_{1} \tilde{+} \rho_{\|}}{\cos \frac{1}{2} \rho_{1}+\rho_{I I} \cos \frac{1}{2} \rho_{1} \sim \rho_{\|}}
\end{aligned}
$$

But the question now naturally arose in D'Alembert's mind, which of these roots belongs to the minimum? or do they both designate minima?

It is difficult to account for that distinguished geometer overlooking the simple and common test furnished by the differential calculus itself for deciding this question: it is even difficult to conceive how the consideration of the problem itself, as presented by the diagram on the globe, should have failed to point out to him the proper interpretation of the twofold result in our last equation: yet such is the case. $\mathrm{He}$ devotes considerable space to proving that the lower sign of our equation does not answer to a minimum twilight. What it does answer to he does not attempt to explain; but he denies that it answers to the maximum.

The general fact, that functions of one independent variable which do not admit of indefinite increase and diminution, can never have two maxima without the intermediate occurrence of a minimum, nor two minima without an intermediate maximum, might have guided him to discover from the figure itself, that the two roots could not both indicate minima ; but rather that one of them necessarily indicated the time of maximum twilight. However, the common appeal to the second differential coefficient determines it at once. For differentiating (6), and inserting the values of $\cos \rho$ given in (7) in the equation, we have for the test the value of

$$
\begin{gathered}
\cos \lambda \frac{1+\cos p_{1} \cos \rho_{\|}}{\cos \rho_{1}+\cos \rho_{\mu}} \mp \cos \lambda \frac{\sin \rho_{1} \sin \rho_{\mu}}{\cos \rho_{1}+\cos \rho_{\mu \prime}} \\
-\cos \lambda \frac{1+\cos \rho_{1} \cos \rho_{\| \prime}}{\cos \rho_{1}+\cos \rho_{H}}
\end{gathered}
$$

the first and third terms of which cancel, and leave, for the test, the sign of

$$
\mp \cos \lambda \frac{\sin \rho_{1} \sin \rho_{\mu}}{\cos \rho_{1}+\cos \rho_{\mu}}
$$

We thus see that the problem has never yet been fully solved, 
Rev. J. Challis on the Theory of the Ball-Pendulum. 185

since the determination of the minimum depends not alone upon the choice of the signs + or - in the equation (7), but also upon the values of $\lambda_{1} \rho$, and $\rho_{\| 1}$. The way in which this takes place is too obvious to need any further remark.

The interpretation, therefore, of Bernoulli's equation is completed and the problem at the same time resolved in the most general case that can be proposed on a spherical earth, and with a uniform declination for the period of twilight on the day in question. It might hence seem that the inquiry, in reference to everything of value which it can afford, might be properly concluded here. Nevertheless, as the curious properties which Delambre has deduced, both in his Astronomie and in his Histoire de l'Astronomie, for the particular case he considered, have in several instances remarkable analogies in the more general one above discussed, it will not be out of place to annex a few of them to the preceding investigation.

['o be continued.]

XXXIV. Theory of the Correction to be applied to a BallPendulum for the Reduction to a Vacuum. By the Rev. J. Chaldis, Fellow of the Cambridge Philosophical Society*.

I $\mathrm{N}$ a previous communication respecting the resistance to the motion of small spherical bodies in elastic fluidst, I attempted to explain, entirely from theoretical considerations, the manner in which the air is acted upon, when a pendulum consisting of a small spherical ball suspended by a very slender wire, performs very small oscillations in it; but I omitted to enter upon any calculation to ascertain the numerical value of the correction required for reducing the time of vibration in air to that in a vacuum. As the theory there advanced is competent to obtain such a result without the aid of experiment, I propose to make this the object of the present communication.

The following equation was obtained in the paper referred to :-

$$
\mathbf{M} v^{2}+m v^{2}=2 g(\mathbf{M}-\mu)(h-x),
$$

in which $M$ is the mass of the ball, $v$ the velocity of its centre, $\mu$ the mass of an equal volume of air, $g$ the force of gravity, $h-x$ the vertical descent of the centre of the ball. The equation without the term $m v^{2}$, is that which was formerly em-

* Communicated by the Author.

† See Lond. and Edinb. Phil. Mag. vol. i. p. 40.

Third Series. Vol. 3. No. 15. Sept. 1833. 